\title{
Postpartum Obstetric Septic Embolism
}

National Cancer Institute

\section{Source}

National Cancer Institute. Postpartum Obstetric Septic Embolism. NCI Thesaurus. Code C111861.

Presence of bacterially-infected tissue in the maternal vascular system which originated from a distant site during the postpartum period. 